\title{
CARCINOMA RICO EM LIPÍDIOS MAMÁRIO, METASTATIZANTE, EM UM \\ CANINO - RELATO DE CASO
}

\author{
CONTE, Fernanda ${ }^{1}$; \\ SERENA, Guilherme Carvalho ${ }^{2}$; \\ RAMOS, Adriano Tony ${ }^{3}$; \\ ZIMERMANN, Francielli Cordeiro ${ }^{3}$; \\ GAMBA, Conrado de Oliveira ${ }^{4}$.
}

Recebido: $10 / 07 / 2018$

Aceito: 18/12/2018

${ }^{1}$ Acadêmica do curso de Medicina Veterinária da Universidade Federal de Santa Catarina (UFSC); ${ }^{2}$ Professor substituto do Curso de Medicina Veterinária/UFSC; ${ }^{3}$ Professores efetivos do Curso de Medicina Veterinária/UFSC; ${ }^{4}$ Professor efetivo (categoria Assistente) do Curso de Medicina Veterinária UNOESC.

\section{RESUMO}

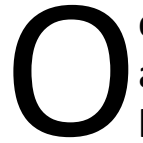

carcinoma rico em lipídios (CRL) é um tipo de neoplasia mamária maligna canina atípica pertencente aos carcinomas especiais. Este relato teve por objetivo descrever lesões macroscópicas e microscópicas de um CRL mamário em um cão. No exame post mortem foram encontrados múltiplos nódulos bilaterais de consistência firme nas mamas torácicas e abdominais. Metástases pulmonares foram observadas. À luz da histopatologia, os nódulos encontrados eram formados por células arranjadas em ninhos sólidos com citoplasma extensamente vacuolizado. As alterações anatomopatológicas descritas são compatíveis com um carcinoma rico em lipídeos mamário canino metastatizante.

Palavras-chave: Cão. Neoplasias mamárias. Carcinomas de tipos especiais. Metástase. 


\section{INTRODUÇÃO}

As neoplasias mamárias atingem cerca de $50 \%$ das cadelas diagnosticadas com tumores (MOTTA, 2008; NÓBREGA, 2013). Em cães, a explicação para sua manifestação corporal pode estar vinculada principalmente ao uso de anticoncepcionais, idade avançada e, em certos casos, à predisposição racial (MOTTA, 2008).

De acordo com a literatura, de 41 a 53\% dos tumores mamários caninos são malignos (BRODY et al., 1983), sendo 90\% deles carcinomas (GILBERTSON et al., 1983). Carcinomas mamários são classificados como: carcinomas ductais in situ, carcinomas em tumores mistos, carcinomas tubulares, carcinomas papilares e carcinomas sólidos. Encontram-se, ainda, tipos especiais de carcinomas, como é o caso do carcinoma de células escamosas e carcinoma rico em lipídios (CASSALI et al., 2014; MAUÉS et al., 2016). O carcinoma rico em lipídios (CRL) é um subtipo de neoplasia mamária maligna, de ocorrência atípica, tanto na oncologia veterinária como na humana. Em animais, pode-se encontrar relato da ocorrência desse tumor em caninos, felinos, hamsters e coelhos (BAUM; HEWICKER-TRAUTWEIN, 2015; CASSALI, 2017; KAMSTOCK et al., 2005; YOSHIMURA et al., 2010).

$\mathrm{Na}$ histopatologia, o CRL caracteriza-se por células distribuídas na forma de cordões, que apresentam em seu citoplasma grande quantidade de vacúolos lipídicos neutros (KAMSTOCK et al., 2005). Estudos relacionados ao CRL evidenciam que este conteúdo lipídico é produzido pelas células neoplásicas (TEl et al., 2012). O diagnóstico preciso é confirmado quando o número de células neoplásicas chega a $80 \%$ do total celular e o diagnóstico diferencial para outros tipos de carcinomas, como os carcinomas ricos em glicogênio, pode ocorrer através da utilização de colorações histológicas especiais, como o SUDAN III e IV (CASSALI et al., 2014).

Diante da infrequência desse tumor, o objetivo deste trabalho foi relatar e descrever um caso de carcinoma rico em lipídios em um canino, evidenciando os achados patológicos que levaram ao diagnóstico tumoral, e também auxiliar o acervo bibliográfico com novas informações sobre essa neoplasia mamária. 


\section{RELATO DE CASO}

Um cão, fêmea, raça Shih Tzu, de 14 anos, apresentou múltiplos nódulos mamários. Segundo o relato do responsável, os nódulos haviam sido identificados há oito meses. O animal foi levado para atendimento clínico com queixa de diarreia e vômito e veio a óbito alguns dias após o atendimento. O cadáver foi encaminhado para o Laboratório de Patologia Veterinária (LABOPAVE) da Universidade Federal de Santa Catarina (UFSC) para a realização do exame post mortem. À necropsia, o exame externo revelou condição corporal ruim. Na cadeia mamária, foram encontrados múltiplos nódulos de consistência firme, brancacentos, os quais variaram de 0,5 a $4 \mathrm{~cm}$. Estavam localizados: no lado esquerdo, nas mamas torácicas cranial e caudal e abdominais cranial e caudal; no lado direito, na mama torácica caudal e abdominal cranial.

O coração apresentava degeneração mixomatosa na válvula mitral. O pulmão estava aderido ao diafragma e revelou bordos abaulados. Além disso, foram encontrados múltiplos nódulos brancacentos variando de 0,3 a $5 \mathrm{~cm}$ com vasos superficialmente proeminentes. Alguns desses nódulos revelaram área de depressão central, dando à lesão aspecto "umbilicado" (Figura 1). Ao corte, os nódulos revelaram-se bem delimitados, difusamente esbranquiçados e, por vezes, apresentaram área periférica de consistência rígida associada a área central de consistência amolecida.

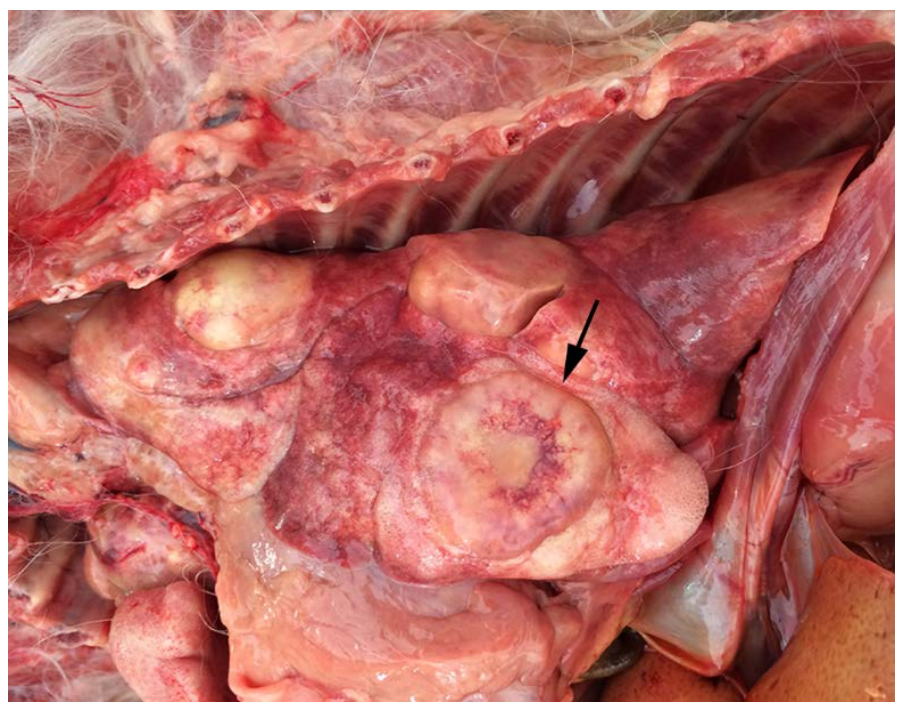

Figura 1 - Canino, Pulmão, CRL metastático representado por inúmeros nódulos com depressão central (aspecto "umbilicado") observados na superfície pleural (seta). 
O fígado apresentou padrão lobular evidente e um nódulo tumoral no lobo medial direito, de coloração branco-amarelada, medindo $10 \times 8 \mathrm{~cm}$. Na pelve renal foram encontrados urólitos de superfície irregular e coloração amarelo escura, com tamanho de aproximadamente $0,4 \mathrm{~cm}$. O útero apresentava conteúdo purulento, sugestivo de piometra, associado à hiperplasia endometrial cística. Uma das adrenais apresentou cortical com aspecto irregular. Após análise macroscópica, fragmentos dos órgãos foram fixados em formol 10\%, clivados, processados por técnica histológica, incluídos em parafina, seccionados e corados por hematoxilina e eosina (HE) para a obtenção das lâminas histológicas.

$\mathrm{Na}$ avaliação histopatológica da glândula mamária foram observados nódulos, formados por células arranjadas em ninhos sólidos, envolvidos por quantidade moderada de estroma. As células apresentavam pleomorfismo moderado, citoplasma vacuolizado ao redor do núcleo que, por vezes, apresentava-se rechaçado para a periferia (células em anel de sinete) (Figura 2). Foram observados 10 campos de maior aumento (obj. 40X) para avaliação do índice mitótico do neoplasma, no qual observaram-se, em média, cinco mitoses por campo. 0 pulmão e o fígado continham nódulos formados por células morfologicamente semelhantes às do tumor primário, associados a áreas de necrose. No sistema urogenital, os glomérulos renais demonstravam glomerulonefrite membranosa e os vasos sanguíneos denotavam congestão. No sistema endócrino, a adrenal continha área de hiperplasia nodular em região capsular. 


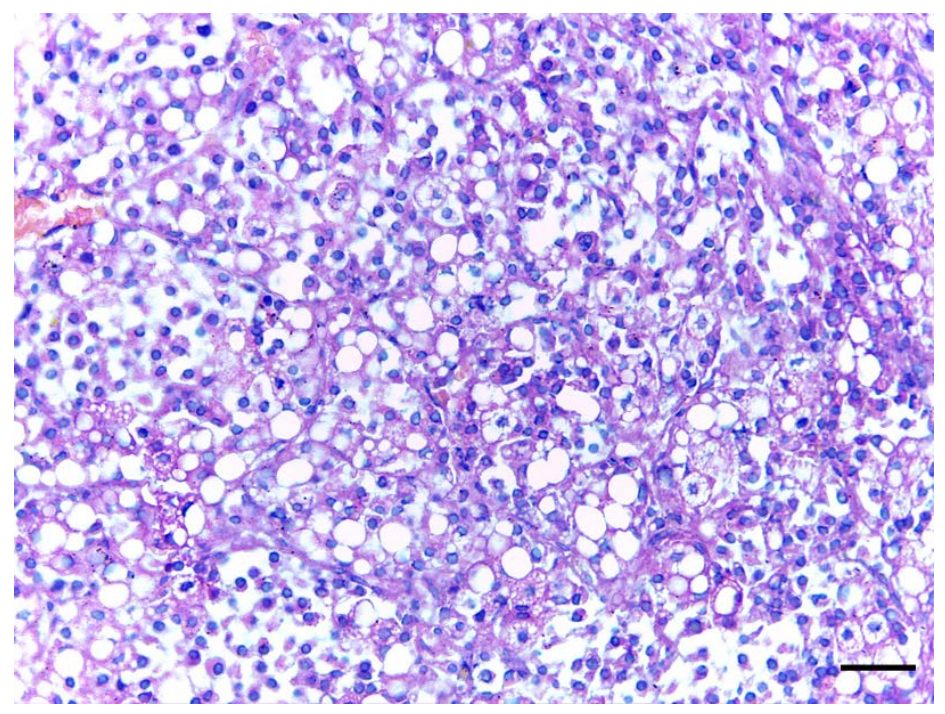

Figura 2 - Canino, glândula mamária, análise histopatológica do tumor primário, revelando múltiplas células moderadamente pleomórficas contendo macrovacúolos intracitoplasmáticos (células em anel de sinete). Coloração por hematoxilina e eosina. Barra $30 \mu \mathrm{m}$. Aumento de 400X.

\section{RESULTADOS E DISCUSSÃO}

O carcinoma rico em lipídios é um subtipo especial de carcinoma mamário com rara ocorrência em animais. Casos de CRL foram descritos em gatos, coelhos e hamsters (BAUM; HEWICKER-TRAUTWEIN, 2015; GAL et al., 2017; KAMSTOCK et al., 2005; YOSHIMURA et al., 2010). Na espécie canina, relatos de casos de CRL na glândula mamária também tem sido descritos (KIM et al., 2014; MAGALHÃES et al., 2012; MONTEROS et al., 2003; PÉREZMARTINEZ et al., 2005; TEl et al., 2012), porém, como se trata de uma variante de carcinomas de rara ocorrência, muitos aspectos patológicos e biológicos ainda devem ser esclarecidos.

As características macroscópicas descritas no CRL canino estão compatíveis com os achados anatomopatológicos relatados na literatura, como: nodulações, únicas ou múltiplas, que podem variar de tamanho; consistência, oscilando de firme à endurecida, que pode ter em sua superfície áreas ulcerativas, necróticas e hemorrágicas. A coloração da massa neoplásica pode apresentar variações entre brancacentas a amareladas (MONTEROS et al., 2003, YOSHIMURA et al., 2010).

O complexo hiperplasia endometrial cística (CHEC) é uma alteração patológica uterina que atinge gatas e cadelas, sendo responsável por ocasionar mudanças nas concentrações 
hormonais de estrógeno e progesterona. Dentre outras funções, esses hormônios estão relacionados com o desenvolvimento e crescimento da glândula mamária, de acordo com os níveis hormonais que se acentuam na puberdade. Dessa forma, o CHEC é considerado um dos fatores que predispõem a neoplasias mamárias, piometra, hidrometra e mucometra (OLIVEIRA, 2007).

Em relação às características histopatológicas do CRL em cães, Monteros et al. (2003) e Goldschmidt et al. (2011), associaram essa neoplasia a células arranjadas em ninhos sólidos, separadas por uma moderada quantidade de estroma, com citoplasma vacuolizado e com núcleo deslocado para a periferia (células em anel de sinete). Essas características são semelhantes ao CRL descrito no presente relato.

De acordo com a literatura, CRL estão frequentemente associados com a ocorrência de metástases no fígado, pulmão e linfonodos regionais na espécie canina. No presente relato de caso, a característica metastática foi observada no pulmão e no fígado, que apresentaram células neoplásicas com características morfológicas semelhantes às encontradas nos tumores primários. Essas metástases, aliadas ao índice mitótico elevado, indicam o comportamento biológico agressivo do neoplasma, neste caso sendo responsáveis pelo óbito do animal. Em estudo realizado por Monteros et al. (2003) foram descritas metástases em cinco dos sete cães relatados, atingindo pulmões e linfonodo inguinal. Em contrapartida, Magalhães et al. (2012) relataram um caso de CRL canino sem metástase.

\section{CONCLUSÃO}

Os achados macroscópicos e microscópicos descritos apontam o diagnóstico de CRL mamário canino, tanto no sítio primário, quanto nos sítios metastáticos. Este relato demonstrou que em cães esta neoplasia pode ter um comportamento biológico altamente metastatizante, podendo levar a complicações clínicas e, consequentemente, ao óbito do animal portador do neoplasma. Novos relatos na literatura poderão auxiliar no diagnóstico precoce de neoplasias suspeitas dessa classe especial de carcinoma, incrementando assim, as possibilidades de tratamento. 


\section{MAMMARY METASTATIC LIPID-RICH CARCINOMA IN A DOG - CASE REPORT}

\section{ABSTRACT}

$\mathrm{T}$

he lipid-rich carcinoma (LRC) is an atypical canine malignant mammary gland neoplasm belonging to the special carcinomas. This report aims to describe the macroscopic and microscopic lesions of a mammary lipid-rich carcinoma in a dog. On the post mortem examination, multiple bilateral nodules firm in consistency were found in the thoracic and abdominal mammary glands. Pulmonary metastases were observed. At histopathology, the nodules were formed by cells arranged in solid nests with extensively vacuolized cytoplasm. The anatomopathological changes described are compatible with a canine mammary metastatic lipid-rich carcinoma.

Keywords: Dog. Mammary neoplasms. Carcinomas of special type. Metastasis.

\section{CARCINOMA RICO EN LIPIDIOS MAMÁRIO CANINO METASTATIZANTE - RELATO DE CASO}

\section{RESUMEN}

$\mathrm{E}$ carcinoma rico en lípidos (CRL) es un tipo de neoplasia mamaria maligna canina atípica perteneciente a los carcinomas especiales. Este relato tiene por objetivo describir las lesiones macroscópicas y microscópicas de un CRL mamario en un perro. En el examen post mortem se encontraron múltiples nódulos bilaterales de consistencia firme, en las mamas torácicas y abdominales. Se observaron metástasis pulmonares. En el caso de los nódulos encontrados, se formaron por células dispuestas en nidos sólidos con citoplasma extensamente vacuolizado. Las alteraciones anatomopatológicas descritas son compatibles con un carcinoma rico en lípidos mamario canino metastatizante.

Palabras clave: Perro. Neoplasias de la mama. Carcinomas de tipos especiales. Metástasis. 


\section{REFERÊNCIAS}

BAUM, B.; HEWICKER-TRAUTWEIN, M. Classification and epidemiology of mammary tumours in pet rabbits (Oryctolagus cuniculus). Journal of Comparative Pathology, v. 152, n. 4, p. 291-298, 2015.

BRODY, R. S.; GOLDSCHMIDT, M. H.; ROSZEL, J. R. Canine mammary gland neoplasms. Journal of the American Animal Hospital Association, v. 19, p. 61-69, 1983.

CASSALI, G. D.; LAVALLE, G. E.; FERREIRA, E.; et al. Consensus for the diagnosis, prognosis and treatment of canine mammary tumors-2013. Brazilian Journal of Veterinary Pathology, v. 7, n. 2, p. 38-69, 2014.

CASSALI, G. D. Patologia Mamária Canina - Do Diagnóstico ao Tratamento. São Paulo: MedVet livros, 2017. 224p.

GAL, A. F.; CĂTOI, C.; TAULESCU, M. A.; et al. Metastatic Lipid-Rich Carcinoma of the Mammary Gland in a Female Cat: Clinicopathological, Histopathological and Immunohistochemical Features. Bulletin UASVM Veterinary Medicine, v. 74, n. 2, p. 156161, 2017.

GILBERTSON, S. R.; KURZMAN, I. D.; ZACHRAU, R. E.; et al. Canine mammary epithelial neoplasms: biologic implications of morphologic characteristics assessed in 232 dogs. Veterinary Pathology, v. 20, n. 2, p. 127-142, 1983.

GOLDSCHMIDT, M.; PEÑA, L.; RASOTTO, R.; et al. Classification and grading of canine mammary tumors. Veterinary pathology, v. 48, n. 1, p. 117-131, 2011.

KAMSTOCK, D. A.; FREDRICKSON, R.; EHRHART, E. J. Lipid-rich carcinoma of the mammary gland in a cat. Veterinary pathology, v. 42, n. 3, p. 360-362, 2005.

KIM, M-C.; KIM, S-Y.; LEE, S-H.; et al. Metastatic lipid-rich mammary carcinoma in a dog. Korean Journal of Veterinary Research, v. 54, n. 4, p. 265-268, 2014.

MAGALHÃES, G. M.; GAVA, F. N.; VOORWALD, F. A.; et al. Carcinoma rico em lipídios em cadela. PUBVET, v. 6, n. 6, p. 1297-2012, 2012.

MAUÉS, T.; FERREIRA, M. D. L. G.; FERREIRA, A. M. R.; et al. Avaliação histopatológica dos linfonodos axilares e inguinais superficiais em cadelas (Canis familiaris) submetidas à mastectomia terapêutica. Veterinária e Zootecnia, v. 23, n. 3, p. 419-429, 2016.

MONTEROS, A. E.; HELLMÉN, E.; RAMÍREZ, G. A.; et al. Lipid-rich carcinomas of the mammary gland in seven dogs: clinicopathologic and immunohistochemical features. Veterinary pathology, v. 40, n. 6, p. 718-723, 2003. 
MOTTA, F. R. Imunorreatividade da prostaglandina E2 relacionada a classificação histopatológica, estadiamento clínico e prognóstico de neoplasias mamárias em cadelas. Jaboticabal: UNESP, 2008. 63p. Dissertação (Mestrado em Cirurgia Veterinária), Faculdade de Ciências Agrárias e Veterinárias, Universidade Estadual Paulista, 2008.

NÓBREGA, D. F. Análise comparativa do diagnóstico citopatológico e histopatológico de neoplasias mamárias em cadelas. Araçatuba: UNESP, 2013. 20p. Monografia (Trabalho de Conclusão de Curso), Universidade Estadual Paulista, 2013.

OLIVEIRA, K. S. Complexo hiperplasia endometrial cística. Acta Scientiae Veterinariae, v. 35, supl. 2, p. s270-s272, 2007.

PÉREZ-MARTÍNEZ, C.; GARCÍA-IGLESIAS, M. J.; DURÁN-NAVARRETE, A. J.; et al. Histopathological and immunohistochemical characteristics of two canine lipid-rich mammary carcinomas. Journal of veterinary medicine. A, Physiology, pathology, clinical medicine, v. 52, n. 2, p. 61-66, 2005.

TEI, M.; UCHIDA, K.; CHAMBERS, J. K.; et al. Mammary Lipid-Rich Carcinoma with Extensive Amyloid Deposition in a Dog. The Japanese Society of Veterinary Science, v. 74, n. 6, p. 809811, 2012.

YOSHIMURA, H.; KIMURA, N.; NAKAHIRA, R.; et al. Lipid-rich carcinoma in the mammary gland of a Djungarian hamster (Phodopus sungorus). Journal of Veterinary Diagnostic Investigation, v. 22, n. 2, p. 305-309, 2010. 